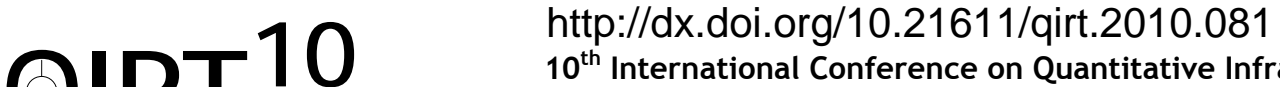 \\ $10^{\text {th }}$ International Conference on Quantitative InfraRed Thermography \\ July 27-30, 2010, Québec (Canada)
}

\section{Towards the use of passive and active infrared thermography to inspect metallic components in the mechanical industry}

\author{
by S. MAILLARD*, J. CADITH*, D.ESCHIMESE*, H. WALASZEK*, \\ H. MOOSHOFER ${ }^{\star \star}$, J.C. CANDORE*** and J.L. BODNAR ${ }^{\star \star \star}$ \\ * CETIM - EPI, Ingénierie de I'Instrumentation, 52, avenue Félix Louat, 60300 Senlis (France), \\ samuel.maillard@cetim.fr \\ ** Siemens AG, Corporate Research and Technologies, GTF Nondestructive Evaluation, CT T DE HW 2, Otto- \\ Hahn-Ring 6, 81739 München (Germany), hubert.mooshofer@siemens.com \\ *** GRESPI/LEO, UFR Sciences Exactes et Naturelles, BP 1039, 51687 Reims cedex 02 (France), jl.bodnar@univ- \\ reims.fr
}

\begin{abstract}
More and more, customer claims a $100 \%$ quality control of the production. In order to take up this challenge, metal forming industries (stamping, forging ...) are especially interested in new non destructive techniques that allow to inspect their parts more rapidly, in a more sustainable way and with costs in accordance with industrial constraints. In collaboration with the University of Reims and Siemens AG, CETIM has then initiated a few years ago projects involving thermal imaging. This paper deals with a comparison between conventional NDE methods such as penetrant testing, magnetic particle inspection or vision with thermal images obtained in passive or active thermography.
\end{abstract}

\section{Introduction}

More and more, customer claims a $100 \%$ quality control of the production. This leads manufacturers to carry out more and more advanced non destructive testing strategies. In order to help the industrialists to take up this challenge, CETIM (French technical center for mechanical Industry) performs a permanent technological survey in the NDT area and investigates how to transfer the most interesting methods to the French mechanics manufactures. Metal forming industries (stamping, forging ...) are especially interested in new non destructive techniques that allow the inspection of their parts more rapidly, in a more sustainable way and with costs in accordance with industrial constraints.

As recent evolutions in the infrared thermographic technology seemed to be of particular interest for such tasks, CETIM has initiated a few years ago many projects involving thermal imaging [1]. The equipment improvements enlarged the capability of passive measurements for certain surface defect detection whereas the developments into active thermography allow the detection of a larger range of defects (small ones on the surface, closed to the surface - nearest or deeper).

In the field of a collaboration with the University of Reims (France) and Siemens AG (Germany), several experiments have been achieved in order to evaluate which kind of thermography (passive or active; pulsed, continuous wave, ...) and which excitation (halogen, laser, flash, induction, ...) was more suitable to detect and characterize defects on metallic components encountered in the mechanical industry.

This paper deals with these main results obtained for crack, opened or not, detection. Thermal images are compared to conventional NDE methods such as penetrant testing or magnetic particle inspection. The possible use of thermography for an on-line inspection is also discussed considering the kind of defects that could be detected and the compatibility between the production rate and the inspection duration.

\section{Application of passive thermography to stamped parts}

In the automotive industry, stamping is a very common process where parts are produced by forming metallic sheet inside a tool. During the process, different kind of defect can be generated on the part. Thus, the process is based on the plastic deformation of the material. But sometimes, variation in the production conditions (material, lubricant, rate) might lead to a local increase of strain in the material and cracks (opened or not) might appear. In some other cases, scraps created during the process are not correctly evacuated in the tool and are crushed on the next strike of the press. They might lead to 
a defect on the surface of the part. As customer claim a 0ppm defect production, industrialists are very keen on nondestructive methods that help them to survey their production.

Classical vision systems are currently used in this industry to check holes or components presence, to control color of the part or to achieve dimensional measurement. But there is no technique that clearly allows satisfactorily detection, on line, of cracks or scraps effect on a wide part of stamped parts.

In this section, potential of passive thermography to detect such defects is evaluated on two kind of parts.

\subsection{Detection of non-opened crack}

The first component presented here is made of conventional coated steel sheet $1,30 \mathrm{~mm}$ thick. In the corner of the part where strains are the most important during stamping, crack may appear and, due to the final function of this component, parts with closed crack are also rejected by the customer. In order to help the operator to detect such defects, several NDE methods (such as acoustic emission, vision inspection) were evaluated.

Thermal images were also achieved on compliant and on non-compliant parts. They were compared with images of the same parts obtained in the visible range. Examples are given in the figure 1.

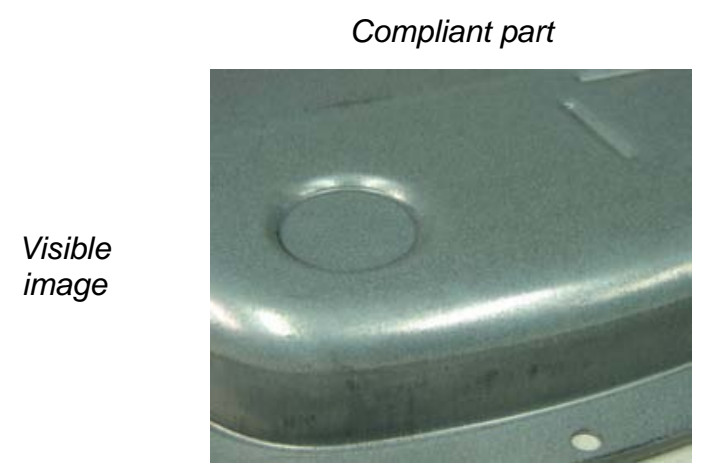

(a)

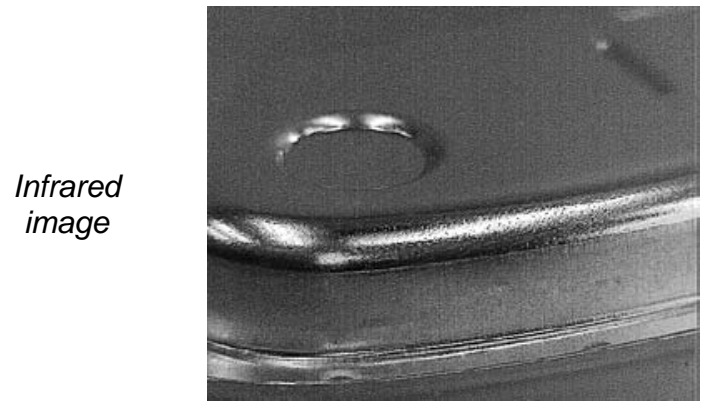

(c)

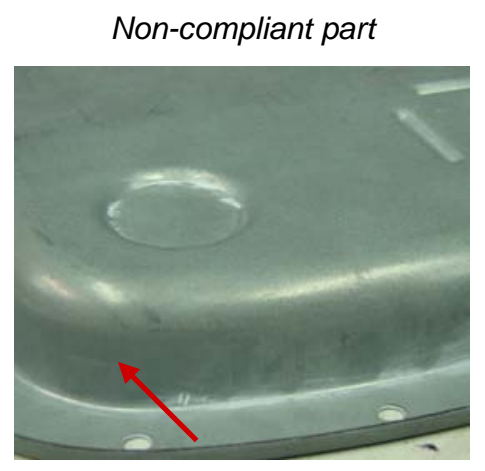

(b)

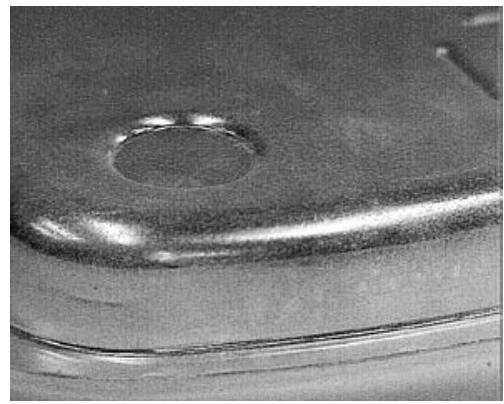

(d)

Fig. 1. Comparison between images obtained in the visible and in the infrared range on stamped parts subjected to closed crack apparition

Image obtained in the visible range slightly highlight the presence of the closed crack but during on-line inspection, the detection is disturbed by the presence of a hot lubricant film on the sample surface. In field measurements have shown that thermal images overcome this drawback. On-line inspection should thus be achieved on such images using conventional algorithms for crack detection.

\subsection{Detection of surface defects}

Parts with surface defects caused by scrap crushed between the forming tool and the metal sheet are also rejected by the costumer. In many cases, conventional vision inspection is used but, for bright metal for examples, the right lighting is very complicated to obtain and such control may not be possible. 
In figure 2, thermal images are compared with images obtained in the visible range on parts with and without surface defect. These parts are made of stainless steel and covered by the lubricant film layed on the metal sheet during the forming process.

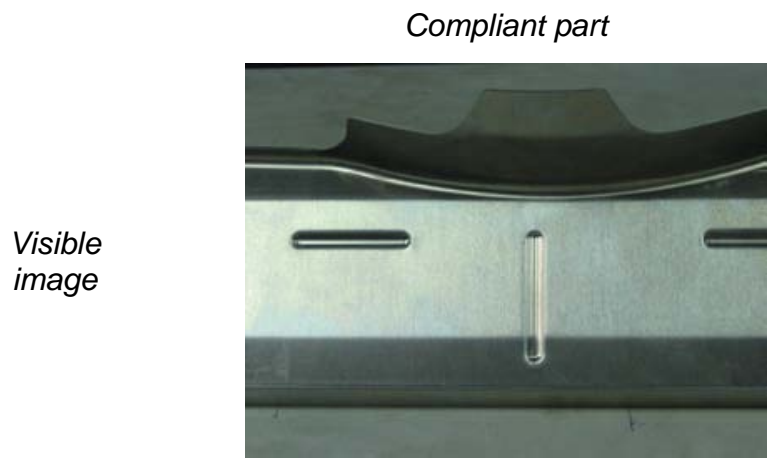

(a)

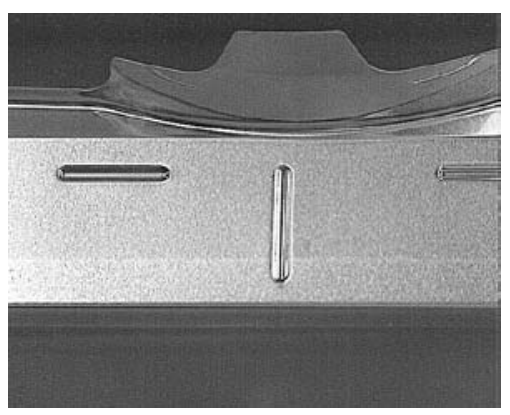

(c)

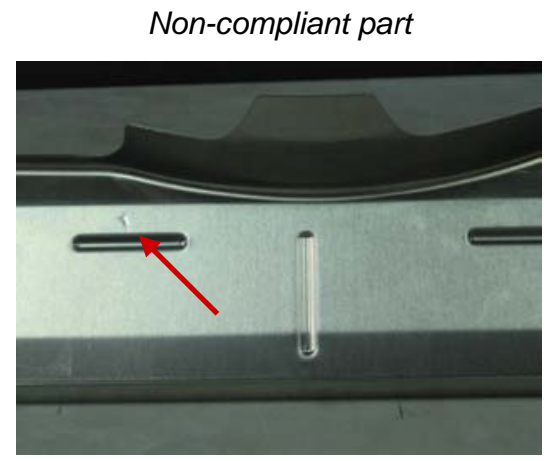

(b)

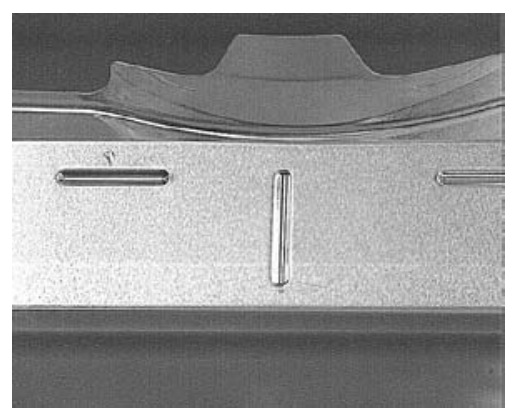

(d)

Fig. 2. Comparison between visible and thermal images on stamped parts subjected to surface defect

Whereas conventional vision inspection is strongly dependant on the lighting for bright material, thermal images enable to detect surface even on material usually not considered for infrared thermography because of their low emissivity (aluminium, polished stainless steel, ...).

\subsection{Towards the use of passive thermography on stamped parts}

The use of passive thermography should be an interesting solution for detection of cracks (opened or not) and surface defects on stamped part. Such measurement is in fact based on the comparison with a reference image and on the detection of abnormal variation of emissivity of the part surface due the presence of the defect. Thus, it could also be applied for material with a low emissivity. In the case of thicker parts $(>\sim 2,00 \mathrm{~mm})$, surface defect due to a scrap could even be detected on the opposite side of the sample. When using such a technique, a particular attention has to be paid to the positioning of the camera, especially to avoid narcissus effect. But compared to vision inspection, the use of infrared waves may avoid problems due to reflection on the sample covered with thin lubricant layer.

\section{Application of active thermography on forged components}

In forging industry, production of parts considered as security components has to be $100 \%$ controlled on $100 \%$ of the surface. Standards thus require to proceed with penetrant testing and magnetic particle inspection. But, industrialists are currently involved in methods that allow the inspection of on line parts in a clean manner. Non Destructive Evaluation is then concerned with the problematic of sustainability and new techniques have to be investigated. Among these, CETIM, through collaboration with the University of Reims and Siemens AG, has initiated research projects involving recent developments in active thermography. 
In this paper, conventional methods were applied on a hub (figure 3.(a)) and on a ball joint (figure 3.(b)). Images are then compared with thermal images obtained through several kind of excitation (laser, flash, induction).

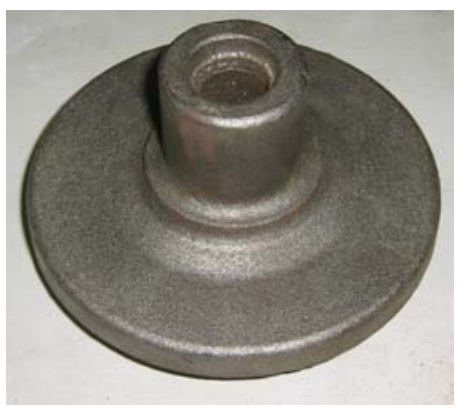

(a) Forged hub with a crack running from the flat surface to the radius

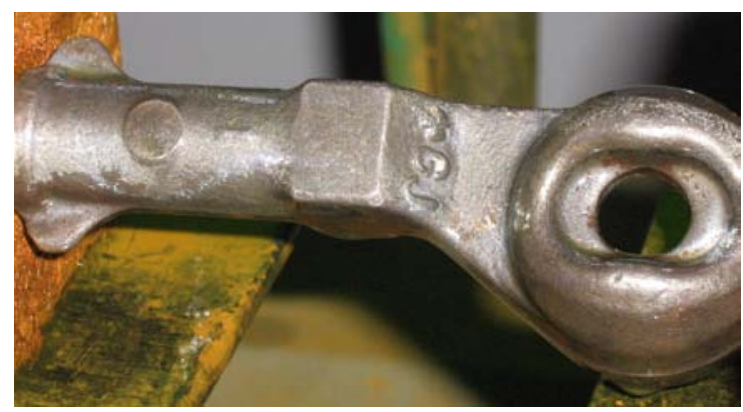

(b) Ball joint

Fig. 3. Photos of the two forged components controlled

\subsection{Detection of a crack on a forged hub}

\subsubsection{By conventional NDE methods}

On the figure 4, penetrant testing as well as magnetic particle inspection images highlight indications that correspond to a crack that goes from the flat surface of the hub and propagates in the fillet radius.

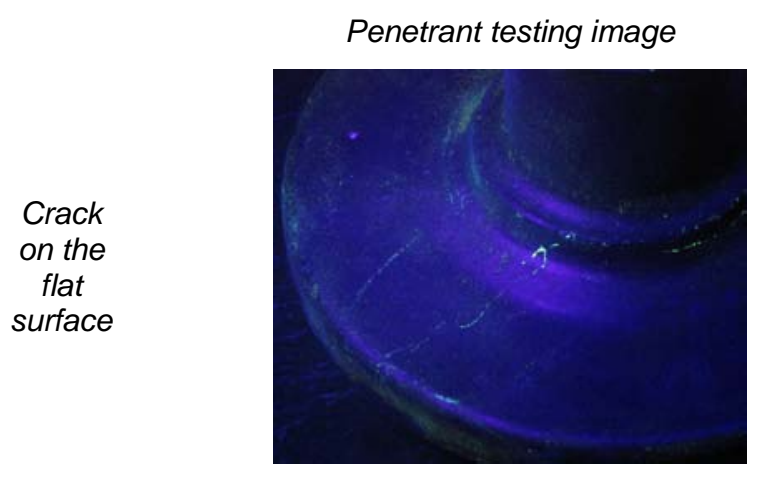

(a)

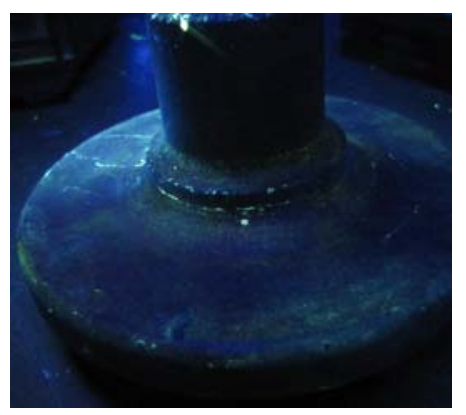

(c)

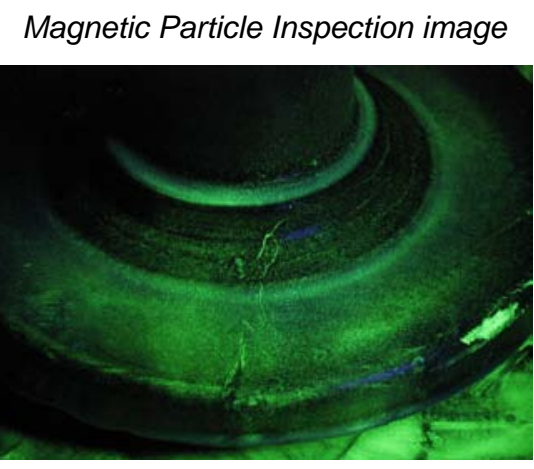

(b)

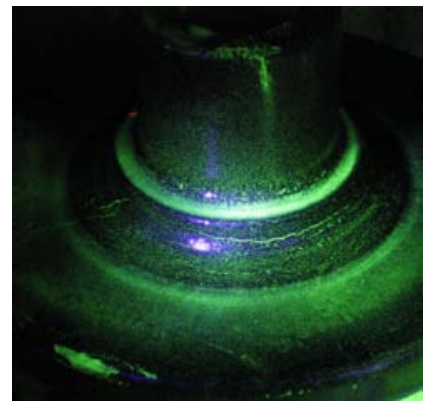

(d)

Fig. 4. Images obtained by penetrant testing and magnetic particle inspection on a cracked forged hub 


\subsubsection{By active thermography}

\subsubsection{Using a laser excitation}

In the 80's, active thermography applications using a laser as excitation source were developed [2,3]. It allowed the detection of open as well as closed crack up to 5,0 mm deep. Such a control was achieved on the forged hub. The reconstructed image is presented on the figure 5 . The observation angle does not allow to observe the crack in the fillet radius but the shape on the flat surface of the indication looks like the one obtained by conventional NDE methods. The inspection rate of such a device is estimated to be about $1,5 \mathrm{~m}^{2} / \mathrm{h}$.

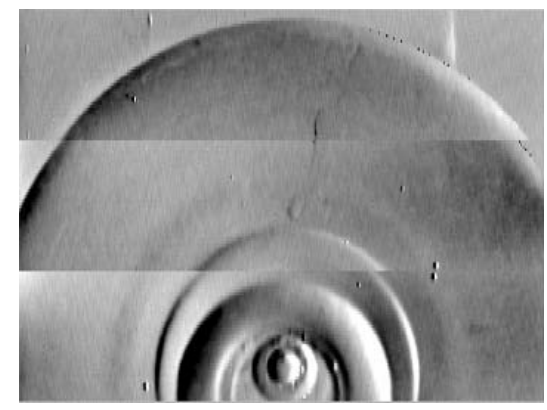

Fig. 5. Thermal image obtained by active thermography using a laser excitation on the flat surface a cracked forged hub

\subsubsection{Using flash excitation}

The same sample was also submitted to a flash excitation $(3,5 \mathrm{~kJ})$. Images obtained at the moment flash are presented in the figure 6 for two different angles.

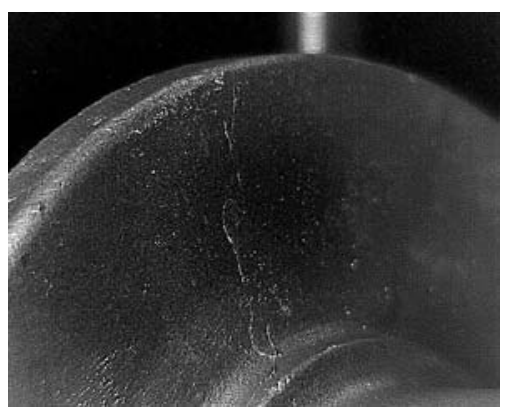

(a) Crack on the flat surface

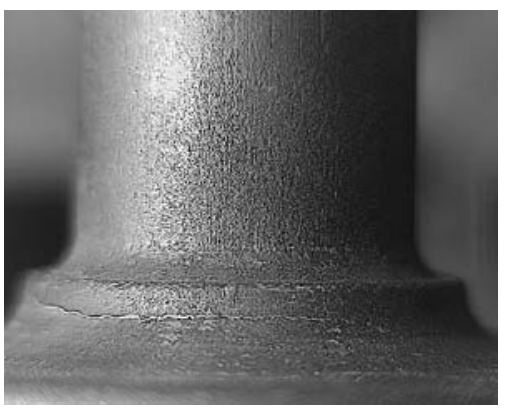

(b) Crack in the fillet radius

Fig. 6. Thermal image obtained by induction thermography on a cracked forged hub

Indications observed in these images are clearly identical to the ones obtained by conventional NDE method and the crack is then properly detected.

\subsubsection{Using induction excitation}

Induction excitation [4], [5] was also used as external heat source on this part. A short Eddy current pulse was induced into the hub at a frequency of about $150 \mathrm{kHz}$, the evolution of the heat distribution was captured with an IR camera and analyzed. Figure 7 corresponds to the phase image obtained. The black line observed on this image is due to the presence of the inductor.

Both indication located on the flat surface and in the fillet radius clearly appear in this image. 


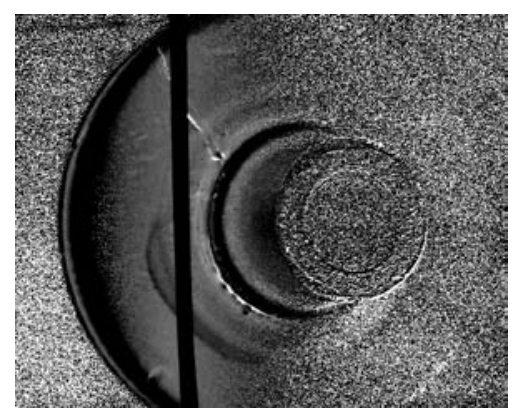

Fig. 7. Phase image obtained by induction thermography on a cracked forged hub

\subsection{Detection of a crack on a forged ball joint}

\subsubsection{By conventional NDE methods}

Magnetic particle inspection highlight a continuous line along the surface of the ball joint. This indication appears, but not so clearly, on the penetrant testing image. It may be because the crack is somewhere closed.

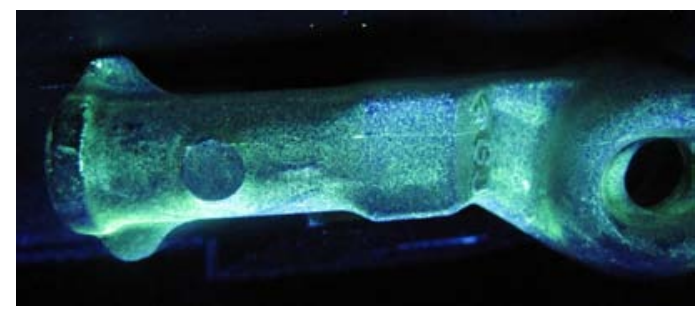

(a) Penetrant testing image

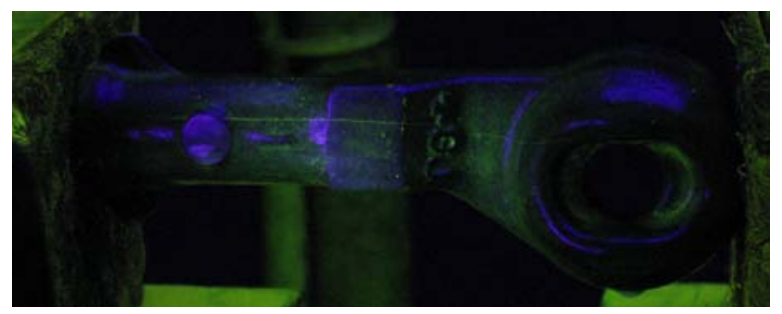

(b) Magnetic Particle Inspection image

Fig. 8. Images obtained by penetrant testing and magnetic particle inspection on a cracked forged ball joint

\subsubsection{By active thermography using a flash excitation}

Thermal image obtained at the moment of the flash excitation is presented on the figure 9. There is no particular indication observed, even when the camera and/or the flash are closer to the analyzed surface or with any observation angle. It then seems that, for such thin defects, limits of the technique are reached.

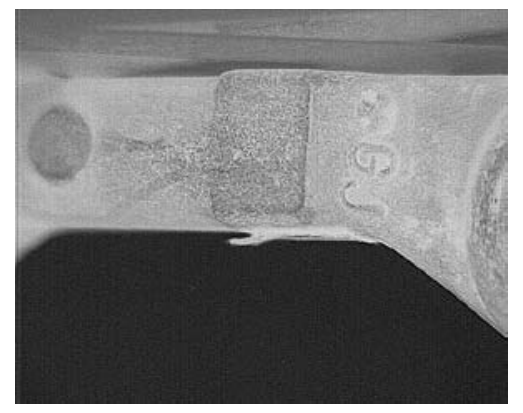

Fig. 9. Thermal image obtained by flash thermography on a cracked forged ball joint 


\subsubsection{By active thermography using induction excitation}

This ball joint was also subject to induction excitation in the same conditions as in 3.1.2.3. Phase image obtained is presented in figure 10. On the excited area (near of the inductor, the black line on the image), the indication corresponding to the crack clearly appear.

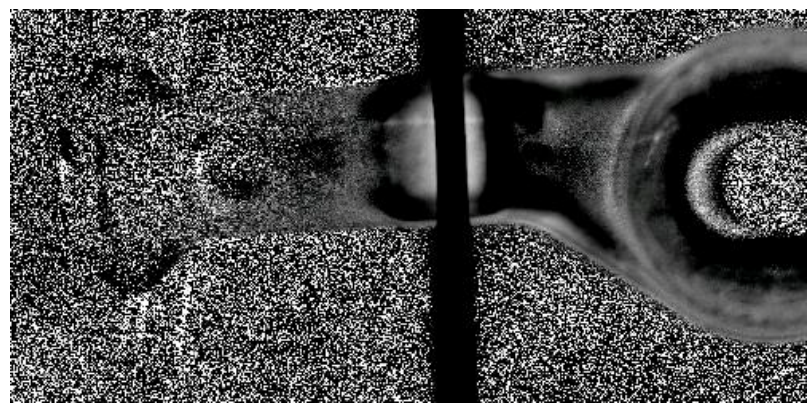

Fig. 10. Phase image obtained by induction thermography on a cracked forged ball joint

\subsection{Towards the use of active thermography on forged parts}

Results presented in this section are encouraging for the development of active thermography for on-line inspection of forged components. One can see that, under certain conditions, active thermography using laser, flash or induction excitation allow to obtain images comparable to the ones obtained using conventional NDE methods. At Siemens, for example, induction thermography is already in use for generator component service [5].

Through these measurements, it seems that laser and induction excitation allow to detect open and closed crack (up to 5,0mm) whereas flash excitation only allow to detect open crack. Flash, which has short life duration regarding the number of samples to inspect, will have to be used in dedicated box to prevent damage for operators. Laser excitation should be the right solution when production rate is not a criterion. Induction excitation is finally of particular interest to inspect productions of parts with repetitive shape where a specific inductor could be designed.

\section{Conclusions}

Recent developments in infrared thermography have led CETIM to investigate new applications on metallic components used in the mechanical industry. Thus, passive thermography should be an interesting tool for crack or surface defect detection on stamped parts whereas active thermography should be of particular interest for forged samples. Configuration of the control (positioning of the camera, excitation source, image processing) has to be carefully chosen according to the considered material, the expected defects (width, length, depth and orientation), the production rate...

Further investigation will be achieved to attempt to detect deeper defects and to automate defect detection from the image sequence. Inductor design will also have to be taken into account in order to improve the use of this technique in the industry. Thus, thermography should appear as a reliable and ecofriendly alternative method for on-line inspection.

\section{REFERENCES}

[1] Maillard S., Cadith J., Walaszek H., Dillenz A., Bodnar J.L., La thermography infrarouge stimulée, une nouvelle technique de contrôle sur les lignes de production ?, COFREND congress proceedings, Toulouse (France), 2008

[2] Bodnar J.L., Edée M., Wear characterization by photothermal radiometry, Wear, Vol. 196, 54-59, 1996

[3] Bodnar J.L., Edée M., Menu C., Besnard R., Le Blanc A., Pigeon M., Sellier J.Y., Cracks detection by a moving photothermal probe, Journal de Physique IV, C7-592, 1994

[4] Vrana J., Goldammer M., Baumann J., Rothenfusser M., Arnold W., Mechanisms and Models for Crack Detection with Induction Thermography, Review of Progress in QNDE 27, pp. 475-482, 2008

[5] Mooshofer H., Goldammer M., Heine W., Rothenfusser M., Bass J., Lombardo E., Vrana J., Induktionsthermographie zur automatischen Prüfung von Generatorkomponenten, DGZfP-Jahrestagung 2009, Münster (Germany), 2009 